\title{
Majorization problems for $p$-valently meromorphic functions of complex order involving certain integral operator
}

\author{
T. Janani and G. Murugusundaramoorthy * \\ School of Advanced Sciences, VIT University, Vellore 632014, Tamilnadu, India \\ *Corresponding author Email: gmsmoorthy@yahoo.com
}

\begin{abstract}
The main object of this paper is to investigate the problem of majorization of certain class of meromorphic p-valent functions of complex order involving certain integral operator. Moreover we point out some new or known consequences of our main result.
\end{abstract}

Keywords: Meromorphic functions, Starlike functions, Convex functions, Majorization problems, Hadamard product (convolution), Integral operator.

2010 AMS Subject Classification:Primary 30C45; Secondary 30C50

\section{Introduction}

Let $f$ and $g$ are analytic functions in the unit disc $\Delta=\{z \in \mathbb{C}:|z|<1\}$. Due to MacGregor [8], (also see [7]) we say that $f$ is majorized by $g$ in $\Delta$ and we write

$$
f(z) \ll g(z),(z \in \Delta)
$$

if there exists a function $\phi$, analytic in $\Delta$, such that

$$
|\phi(z)|<1 \text { and } f(z)=\phi(z) g(z), \quad z \in \Delta .
$$

It may be noted here that (1) is closely related to the concept of quasi-subordination between analytic functions.

Also we say that $f$ is subordinate to $g$ denoted by $f \prec g$ (see [9]), if there exists a Schwarz function $\omega$ which is analytic in $\Delta$ with $\omega(0)=0$ and $|\omega(z)|<1$ for all $z \in \Delta$, such that

$$
f(z)=g(\omega(z)), z \in \Delta .
$$

We denote this subordination by $f \prec g$. Furthermore, if the function $g$ is univalent in $\Delta$, we have

$$
f \prec g \Longleftrightarrow f(0)=g(0) \text { and } f(\Delta) \subset g(\Delta) .
$$

Denote by $\mathcal{S}^{*}(\gamma)$ and $\mathcal{C}(\gamma)$ the class of starlike and convex functions of complex order $\gamma(\gamma \in \mathbb{C} \backslash\{0\})$, satisfying the following conditions

$$
\frac{f(z)}{z} \neq 0 \text { and } \Re\left(1+\frac{1}{\gamma}\left[\frac{z f^{\prime}(z)}{f(z)}-1\right]\right)>0
$$


and

$$
f^{\prime}(z) \neq 0 \text { and } \Re\left(1+\frac{1}{\gamma}\left[\frac{z f^{\prime \prime}(z)}{f^{\prime}(z)}\right]\right)>0,(z \in \Delta)
$$

respectively. Further,

$$
\mathcal{S}^{*}\left((1-\delta) \cos \lambda e^{-i \lambda}\right)=S^{*}(\delta, \lambda),|\lambda|<\frac{\pi}{2} ; 0 \leq \delta \leq 1
$$

the class of $\lambda$ - spiral-like function of order $\delta$ investigated by Libera [4] and

$$
\mathcal{S}^{*}\left(\cos \lambda e^{-i \lambda}\right)=S^{*}(\lambda),|\lambda|<\frac{\pi}{2}
$$

the class of spiral-like functions introduced by Spacek [10]( also see [11]).

A mojorization problem for the class of analytic starlike functions have been investigated by MacGregor [8] and Altintas et al. [1]. Recently Goyal and Goswami [3] extended these results for the class of meromorphic functions making use of certain integral operator.

Let $\Sigma_{p}$ be the class of $p$-valently meromorphic functions which are analytic and univalent in the punctured unit disk

$$
\Delta^{*}=\{z \in \mathbb{C}: 0<|z|<1\}=\Delta \backslash\{0\}
$$

of the form

$$
f(z)=\frac{1}{z^{p}}+\sum_{n=1}^{\infty} a_{n-p} z^{n-p} .
$$

with a simple pole at the origin.

Due to Aqlan et al. [2] (see [5]), we recall the integral operator $\mathcal{J}_{\beta, p}^{\alpha}$ for meromorphic functions $f \in \Sigma_{p}$ as given below,

$$
\begin{gathered}
\mathcal{J}_{\beta, p}^{\alpha}: \Sigma_{p} \rightarrow \Sigma_{p} \\
\mathcal{J}_{\beta, p}^{\alpha} f(z)=\left(\begin{array}{c}
\alpha+\beta-1 \\
\beta-1
\end{array}\right) \frac{1}{z^{p+\beta}} \int_{0}^{z}\left(1-\frac{t}{z}\right)^{\alpha-1} t^{\beta+p-1} f(t) d t \\
\mathcal{J}_{\beta, p}^{\alpha} f(z)= \begin{cases}f(z) & \alpha=0, \beta>-1, p \in \mathbb{N}, f \in \Sigma_{p} \\
\frac{1}{z^{p}}+\frac{\Gamma(\alpha+\beta)}{\Gamma(\beta)} \sum_{n=1}^{\infty} \frac{\Gamma(n+\beta)}{\Gamma(n+\alpha+\beta)} a_{n-p} z^{n-p}, & \alpha>0, \beta>-1, p \in \mathbb{N}, f \in \Sigma_{p} .\end{cases}
\end{gathered}
$$

The following relation for $\mathcal{J}_{\beta, p}^{\alpha} f(z)$ can be obtained by simple calculation,

$$
z\left(\mathcal{J}_{\beta, p}^{\alpha} f(z)\right)^{\prime}=(\alpha+\beta-1) \mathcal{J}_{\beta, p}^{\alpha-1} f(z)-(\alpha+\beta+p-1) \mathcal{J}_{\beta, p}^{\alpha} f(z) .
$$

Using (6), the below recurrence relation for $\mathcal{J}_{\beta, p}^{\alpha} f(z)$ can be obtained easily,

$$
z\left(\mathcal{J}_{\beta, p}^{\alpha} f(z)\right)^{(q+1)}=(\alpha+\beta-1)\left(\mathcal{J}_{\beta, p}^{\alpha-1} f(z)\right)^{(q)}-(\alpha+\beta+p+q-1)\left(\mathcal{J}_{\beta, p}^{\alpha} f(z)\right)^{(q)} .
$$

In the present paper we investigate a majorization problem for the class of $p$-valently meromorphic starlike functions of complex order associated with the generalized integral operator due to Aqlan [2] and Murugusundaramoorthy and Magesh [6].

Definition 1.1. A function $f(z) \in \Sigma_{p}$ is said to in the class $\mathcal{M}_{\alpha, \beta}^{p, q}(\gamma, A, B)$ of meromorphic functions of complex order $\gamma \neq 0$ in $\Delta^{*}$ if and only if

$$
1-\frac{1}{\gamma}\left[\frac{z\left(\mathcal{J}_{\beta, p}^{\alpha} f(z)\right)^{(q+1)}}{\left(\mathcal{J}_{\beta, p}^{\alpha} f(z)\right)^{(q)}}+p+q\right] \prec \frac{1+A z}{1+B z},
$$

where $z \in \Delta^{*}, p, q \in \mathbb{N}_{0}=\mathbb{N} \cup\{0\}, \beta>-1, \alpha>0, \gamma \in \mathbb{C} \backslash\{0\}$ and $-1 \leq B<A \leq 1$. 
For simplicity, we put

$$
\mathcal{M}_{\alpha, \beta}^{p, q}(\gamma, 1,-1)=\mathcal{M}_{\alpha, \beta}^{p, q}(\gamma)
$$

where $\mathcal{M}_{\alpha, \beta}^{p, q}(\gamma)$ denote the class of functions $f \in \Sigma_{p}$ satisfying the following inequality:

$$
\Re\left(1-\frac{1}{\gamma}\left[\frac{z\left(\mathcal{J}_{\beta, p}^{\alpha} f(z)\right)^{(q+1)}}{\left(\mathcal{J}_{\beta, p}^{\alpha} f(z)\right)^{(q)}}+p+q\right]\right)>0
$$

where $z \in \Delta^{*}, p, q \in \mathbb{N}_{0}=\mathbb{N} \cup\{0\}, \beta>-1, \alpha>0, \gamma \in \mathbb{C} \backslash\{0\}$.

Example 1.2. Putting $\gamma=(p-\delta) \cos \lambda e^{-i \lambda},|\lambda|<\frac{\pi}{2} ; 0 \leq \delta<p$ the class

$$
\mathcal{M}_{\alpha, \beta}^{p, q}(\gamma)=\mathcal{M}_{\alpha, \beta}^{p, q}\left((p-\delta) \cos \lambda e^{-i \lambda}\right) \equiv \mathcal{M}_{\alpha, \beta}^{p, q}(\delta, \lambda)
$$

called the generalized class of $\lambda$-spiral-like functions of order $\delta(0 \leq \delta<p)$ if

$$
\Re\left(e^{i \lambda}\left[\frac{z\left(\mathcal{J}_{\beta, p}^{\alpha} f(z)\right)^{(q+1)}}{\left(\mathcal{J}_{\beta, p}^{\alpha} f(z)\right)^{(q)}}+q\right]\right)<-\delta \cos \lambda
$$

where $z \in \Delta^{*}, p, q \in \mathbb{N}_{0}=\mathbb{N} \cup\{0\}, \beta>-1, \alpha>0, \gamma \in \mathbb{C} \backslash\{0\}$.

Example 1.3. Putting $\gamma=(p-\delta) ; 0 \leq \delta<p$ the class $\mathcal{M}_{\alpha, \beta}^{p, q}(p-\delta) \equiv \mathcal{M}_{\alpha, \beta}^{p, q}(\delta)$, the generalized class of $p-$ valently meromorphic starlike functions of order $\delta(0 \leq \delta<p)$ if

$$
\Re\left(\frac{z\left(\mathcal{J}_{\beta, p}^{\alpha} f(z)\right)^{(q+1)}}{\left(\mathcal{J}_{\beta, p}^{\alpha} f(z)\right)^{(q)}}+q\right)<-\delta
$$

where $z \in \Delta^{*}, p, q \in \mathbb{N}_{0}=\mathbb{N} \cup\{0\}, \beta>-1, \alpha>0, \gamma \in \mathbb{C} \backslash\{0\}$.

Remark 1.4. By taking $q=0$ in Example 1.3, $\mathcal{M}_{\alpha, \beta}^{p, 0}(p-\delta) \equiv \mathcal{M}_{\alpha, \beta}^{p}(\delta)$ the class of p-valently meromorphic starlike functions of order $\delta(0 \leq \delta<p)$ if

$$
\Re\left(\frac{z\left(\mathcal{J}_{\beta, p}^{\alpha} f(z)\right)^{\prime}}{\left(\mathcal{J}_{\beta, p}^{\alpha} f(z)\right)}\right)<-\delta
$$

where $z \in \Delta^{*}, \beta>-1, \alpha>0, \gamma \in \mathbb{C} \backslash\{0\}$.

\section{Majorization problem for the class $\mathcal{M}_{\alpha, \beta}^{p, q}(\gamma, A, B)$}

Theorem 2.1. Let the function $f \in \Sigma_{p}$ and $g \in \mathcal{M}_{\alpha, \beta}^{p, q}(\gamma, A, B)$ if $\left(\mathcal{J}_{\beta, p}^{\alpha} f(z)\right)^{(q)}$ is majorized by $\left(\mathcal{J}_{\beta, p}^{\alpha} g(z)\right)^{(q)}$ in $\Delta^{*}$ then

$$
\left|\left(\mathcal{J}_{\beta, p}^{\alpha-1} f(z)\right)^{(q)}\right| \leq\left|\left(\mathcal{J}_{\beta, p}^{\alpha-1} g(z)\right)^{(q)}\right|, \quad|z| \leq r_{1}
$$

$r_{1}=r_{1}(A, B, \alpha, \beta, \gamma, \rho)$ is the smallest positive root of the equation

$$
\begin{aligned}
& |(\alpha+\beta-1) B-\gamma(A-B)| r^{3}-\{(\alpha+\beta-1)+2 \rho|B|\} r^{2} \\
-\quad & \{|(\alpha+\beta-1) B-\gamma(A-B)|+2 \rho\} r+(\alpha+\beta-1)=0,
\end{aligned}
$$

where $z \in \Delta^{*}, p, q \in \mathbb{N}_{0}=\mathbb{N} \cup\{0\}, \beta>-1, \alpha>0, \gamma \in \mathbb{C} \backslash\{0\}$ and $-1 \leq B<A \leq 1$.

Proof. Since $g(z) \in \mathcal{M}_{\alpha, \beta}^{p, q}(\gamma, A, B)$, we readily obtain from (8) that, if

$$
1-\frac{1}{\gamma}\left[\frac{z\left(\mathcal{J}_{\beta, p}^{\alpha} g(z)\right)^{(q+1)}}{\left(\mathcal{J}_{\beta, p}^{\alpha} g(z)\right)^{(q)}}+p+q\right]=\frac{1+A w(z)}{1+B w(z)}
$$

where $w$ denotes the well known class of bounded analytic functions in $\Delta$ and

$$
w(0)=0 \quad \text { and } \quad|w(z)| \leq|z|, \quad(z \in \Delta) .
$$


From (14),we get

$$
\frac{z\left(\mathcal{J}_{\beta, p}^{\alpha} g(z)\right)^{(q+1)}}{\left(\mathcal{J}_{\beta, p}^{\alpha} g(z)\right)^{(q)}}=-\frac{(p+q)+[(p+q) B+\gamma(A-B)] w(z)}{1+B w(z)} .
$$

Using (7) in the above equation, we get,

$$
\left(\mathcal{J}_{\beta, p}^{\alpha} g(z)\right)^{(q)}=\frac{(\alpha+\beta-1)[1+B w(z)]}{(\alpha+\beta-1)+[(\alpha+\beta-1) B-\gamma(A-B)] w(z)}\left(\mathcal{J}_{\beta, p}^{\alpha-1} g(z)\right)^{(q)} .
$$

Hence, by making use of (15), we get,

$$
\left|\left(\mathcal{J}_{\beta, p}^{\alpha} g(z)\right)^{(q)}\right| \leq \frac{(\alpha+\beta-1)[1+|B||z|]}{(\alpha+\beta-1)-|(\alpha+\beta-1) B-\gamma(A-B)||z|}\left|\left(\mathcal{J}_{\beta, p}^{\alpha-1} g(z)\right)^{(q)}\right| .
$$

Since $\left(\mathcal{J}_{\beta, p}^{\alpha} f(z)\right)^{(q)}$ is majorized by $\left(\mathcal{J}_{\beta, p}^{\alpha} g(z)\right)^{(q)}$ in $\Delta^{*}$ from (2), we have

$$
\left(\mathcal{J}_{\beta, p}^{\alpha} f(z)\right)^{(q)}=\phi(z)\left(\mathcal{J}_{\beta, p}^{\alpha} g(z)\right)^{(q)}
$$

Differentiating the above equation w.r.t $z$ and multiplying by $z$, we have,

$$
z\left(\mathcal{J}_{\beta, p}^{\alpha} f(z)\right)^{(q+1)}=z \phi^{\prime}(z)\left(\mathcal{J}_{\beta, p}^{\alpha} g(z)\right)^{(q)}+z \phi(z)\left(\mathcal{J}_{\beta, p}^{\alpha} g(z)\right)^{(q+1)} .
$$

By using (7), we get,

$$
\left(\mathcal{J}_{\beta, p}^{\alpha-1} f(z)\right)^{(q)}=\frac{z}{\alpha+\beta-1} \phi^{\prime}(z)\left(\mathcal{J}_{\beta, p}^{\alpha} g(z)\right)^{(q)}+\phi(z)\left(\mathcal{J}_{\beta, p}^{\alpha-1} g(z)\right)^{(q)} .
$$

Noting that the Schwarz function $\phi(z)$ satisfies

$$
\left|\phi^{\prime}(z)\right| \leq \frac{1-|\phi(z)|^{2}}{1-|z|^{2}}
$$

and using (18) and (20) in (19) we have

$$
\begin{aligned}
& \left|\left(\mathcal{J}_{\beta, p}^{\alpha-1} f(z)\right)^{(q)}\right| \\
& \leq\left(|\phi(z)|+\frac{\left(1-|\phi(z)|^{2}\right)}{\left(1-|z|^{2}\right)} \cdot \frac{|z|(1+|B||z|)}{(\alpha+\beta-1)-|(\alpha+\beta-1) B-\gamma(A-B)||z|}\right)\left|\left(\mathcal{J}_{\beta, p}^{\alpha-1} g(z)\right)^{(q)}\right|
\end{aligned}
$$

which upon setting

$$
|z|=r \text { and }|\phi(z)|=\rho, \quad(0 \leq \rho \leq 1)
$$

leads us to the inequality

$$
\left|\left(\mathcal{J}_{\beta, p}^{\alpha-1} f(z)\right)^{(q)}\right| \leq \frac{\theta(\rho)}{\left(1-r^{2}\right)\{(\alpha+\beta-1)-|(\alpha+\beta-1) B-\gamma(A-B)| r\}}\left|\left(\mathcal{J}_{\beta, p}^{\alpha-1} g(z)\right)^{(q)}\right|,
$$

where

$$
\theta(\rho)=\rho\left(1-r^{2}\right)\{(\alpha+\beta-1)-|(\alpha+\beta-1) B-\gamma(A-B)| r\}+\left(1-\rho^{2}\right)(1+|B| r) r
$$

takes its maximum value at $\rho=1$. Furthermore, if $0 \leq \sigma \leq r_{1}$, the function $\varphi(\rho)$ defined by

$$
\varphi(\rho)=\rho\left(1-\sigma^{2}\right)\{(\alpha+\beta-1)-|(\alpha+\beta-1) B-\gamma(A-B)| \sigma\}+\left(1-\rho^{2}\right)(1+|B| \sigma) \sigma
$$

is an increasing function on $(0 \leq \rho \leq 1)$ so that

$$
\varphi(\rho) \leq \varphi(1)=\left(1-\sigma^{2}\right)\{(\alpha+\beta-1)-|(\alpha+\beta-1) B-\gamma(A-B)| \sigma\} .
$$

Therefore, from this fact, (21) gives the inequality (12). 


\section{Corollaries and Concluding Remarks}

By taking $A=1 ; B=-1$ and $\rho=1$ in Theorem 2.1, we state the following corollary without proof.

Corollary 3.1. Let the function $f \in \Sigma_{p}$ and $g(z) \in \mathcal{M}_{\alpha, \beta}^{p, q}(\gamma)$ if $\left(\mathcal{J}_{\beta, p}^{\alpha} f(z)\right)^{(q)}$ is majorized by $\left(\mathcal{J}_{\beta, p}^{\alpha} g(z)\right)^{(q)}$ in $\Delta^{*}$ then

$$
\left|\left(\mathcal{J}_{\beta, p}^{\alpha-1} f(z)\right)^{(q)}\right| \leq\left|\left(\mathcal{J}_{\beta, p}^{\alpha-1} g(z)\right)^{(q)}\right|, \quad|z| \leq r_{2}
$$

where $r_{2}=r_{2}(\alpha, \beta, \gamma)$ is the smallest positive root of the equation

$$
\begin{aligned}
& \left\{|(\alpha+\beta-1)+2 \gamma| r^{3}-\{\alpha+\beta+1\} r^{2}-\{|(\alpha+\beta-1)+2 \gamma|+2\} r+(\alpha+\beta-1)=0,\right. \text { given by } \\
& r_{2}=\frac{L_{1}-\sqrt{L_{1}^{2}-4|\alpha+\beta-1+2 \gamma|(\alpha+\beta-1)}}{2|\alpha+\beta-1+2 \gamma|}
\end{aligned}
$$

and $L_{1}=\alpha+\beta+1+|\alpha+\beta-1+2 \gamma|$.

By setting $\alpha=1$ in Corollary 3.1, we state the following corollary.

Corollary 3.2. Let the function $f \in \Sigma_{p}$ and $g(z) \in \mathcal{M}_{\alpha, \beta}^{p, q}(\gamma)$ if $\left(\mathcal{J}_{\beta, p}^{1} f(z)\right)^{(q)}$ is majorized by $\left(\mathcal{J}_{\beta, p}^{1} g(z)\right)^{(q)}$ in $\Delta^{*}$ then

$$
\left|(f(z))^{(q)}\right| \leq\left|(g(z))^{(q)}\right|, \quad|z| \leq r_{3},
$$

where $r_{3}=r_{3}(1, \beta, \gamma)$ is the smallest positive root of the equation

$$
\begin{aligned}
& |\beta+2 \gamma| r^{3}-(\beta+2) r^{2}-\{|\beta+2 \gamma|+2\} r+\beta=0, \text { given by } \\
& r_{3}=\frac{L_{2}-\sqrt{L_{2}^{2}-4 \beta|\beta+2 \gamma|}}{2|\beta+2 \gamma|}
\end{aligned}
$$

and $L_{2}=\beta+2+|\beta+2 \gamma|$.

By setting $\alpha=1, \beta=1$ and $\gamma=p-\delta$ in Corollary 3.1, we state the following corollary.

Corollary 3.3. Let the function $f \in \Sigma_{p}$ and $g(z) \in \mathcal{M}_{\alpha, \beta}^{p, q}(\delta)$ if $\left(\mathcal{J}_{1, p}^{1} f(z)\right)^{(q)}$ is majorized by $\left(\mathcal{J}_{1, p}^{1} g(z)\right)^{(q)}$ in $\Delta^{*}$ then

$$
\left|(f(z))^{(q)}\right| \leq\left|(g(z))^{(q)}\right|, \quad|z| \leq r_{4}
$$

where $r_{4}=r_{4}(1,1,(p-\delta) 1)$ is the smallest positive root of the equation

$$
\begin{aligned}
& |1+2(p-\delta)| r^{3}-3 r^{2}-\{|1+2(p-\delta)|+2\} r+1=0, \text { given by } \\
& r_{4}=\frac{L_{3}-\sqrt{L_{3}^{2}-4|1+2(p-\delta)|}}{2|1+2(p-\delta)|}
\end{aligned}
$$

and $L_{3}=3+|1+2(p-\delta)|$.

Remark 3.4. By taking $p=1$ and $q=0$, Corollary 3.3 yields results of Goyal and Gosami[3]. proof.

By taking $\gamma=(p-\delta) \cos \lambda e^{-i \lambda}\left(|\lambda|<\frac{\pi}{2}, \delta(0 \leq \delta<p)\right.$, in Corollary 3.1, we state the following corollary without

Corollary 3.5. Let the function $f \in \Sigma_{p}$ and $g(z) \in \mathcal{M}_{\alpha, \beta}^{p, q}(\alpha, \lambda)$ if $\left(\mathcal{J}_{\beta, p}^{\alpha} f(z)\right)^{(q)}$ is majorized by $\left(\mathcal{J}_{\beta, p}^{\alpha} g(z)\right)^{(q)}$ in $\Delta^{*}$ then

$$
\left|\left(\mathcal{J}_{\beta, p}^{\alpha} f(z)\right)^{(q)}\right| \leq\left|\mathcal{J}_{\beta, p}^{\alpha}(g(z))^{(q)}\right|,|z| \leq r
$$

where $r=r(T, \lambda)$ is given by

$$
r=\frac{T-\sqrt{T^{2}-4\left|\alpha+\beta-1+2(p-\delta) \cos \lambda e^{-i \lambda}\right|(\alpha+\beta-1)}}{2\left|\alpha+\beta-1+2(p-\delta) \cos \lambda e^{-i \lambda}\right|}
$$

and

$$
T=(\alpha+\beta+1)+\left|1+2(p-\delta) \cos \lambda e^{-i \lambda}\right| .
$$

Concluding Remarks: Further specializing the parameters $\alpha, \beta$ one can define the various other interesting subclasses of $\Sigma_{p}$ involving the various integral operators and the corresponding corollaries as mentioned above can be derived easily. 


\section{References}

[1] O. Altintaş, Ö.Özkan and H. M. Srivastava, Majorization by starlike functions of complex order, Complex Variables Theory Appl. 46 (2001), no. 3, 207-218.

[2] E.Aqlan, J.M.Jahangiri, and S.R.Kulkarni, Certain integral operators applied to meromorphic p-valent functions, J. Nat. Geom., 24 (2003),111-120.

[3] S. P. Goyal and P.Goswami , Majorization for certain classes of meromorphic functions defined by integral operator, Annales. Univ. Marie-Curie-Sklod. Math. Lett. 66 (2012), no. 2, 57-62.

[4] R. J. Libera, Univalent a spiral-like functions, Canadian. J. Math. Soc. 19 (1967), 449-456.

[5] A.Y. Lashin, On certain subclasses of meromorphic functions associated with certain integral operators, Comput. Math Appl. 59 (1) (2010), 524-531.

[6] G.Murugusundaramoorthy and N. Magesh, Differential subordination and superordination of integral operator on meromorphic functions", Math. Sci. Res. J. 13 (2009), no. 3, 68-79

[7] Z.Nahari, Conformal mapping, Mac Gra-Hill Book Company, New York; Toronto and London, 1952.

[8] T. H.Mac Gregor, Majorization by univalent functions, Duke Math. J. 34 (1967), 95-102.

[9] S. S. Miller,and P. T.Mocanu, Differential subordinations, Monographs and Textbooks in Pure and Applied Mathematics, 225, Dekker, New York, 2000.

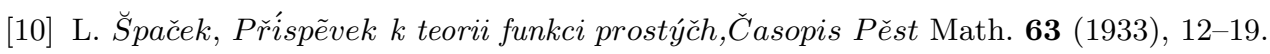

[11] H.M. Srivastava, S.Owa, A note on certain class of spiral-like functions, Rend.Sem.Mat.Univ.Padavo., 80 (1988), 17-24. 